Gut, 1980, 21, 607-613

\title{
Effect of Nissen fundoplication on competence of the gastro-oesophageal junction*
}

\author{
R J R GOODALL AND J G TEMPLE $\dagger$ \\ From the Department of Surgery, Hope Hospital, University of Manchester School of Medicine, Salford
}

SUMMARY The changes produced by the Nissen fundoplication were measured in 12 patients, who required surgery to control their reflux oesophagitis. The gastro-oesophageal junction of each patient was studied before and three months after operation by station pullthrough manometry and prolonged pH monitoring of the distal oesophagus. All patients were free from reflux symptoms post-operatively. The Nissen fundoplication resulted in a significant increase in the pressure, but not the length, of the lower oesophageal high pressure zone. A greater proportion of this zone was situated in the abdomen postoperatively. Prolonged $\mathrm{pH}$ monitoring showed a significant improvement in all the measured indices of acid reflux. Nissen fundoplication restores competence to the gastro-oesophageal junction as judged by manometry and $\mathrm{pH}$ monitoring. This kind of study should be performed to document the efficiency of other anti-reflux procedures.

Although the precise nature of the mechanism which prevents abnormal reflux of gastric contents into the lower oesophagus is not clearly defined and may be dependent upon more than one factor, ${ }^{123}$ it is now well recognised that failure of this mechanism may occur independently of the anatomical abnormality of a sliding hiatal hernia. ${ }^{45}$ Consequent upon this understanding of the pathophysiology of gastro-oesophageal reflux has come the reappraisal of the aim of surgery in the treatment of those patients whose symptoms and oesophagitis are unresponsive to conservative therapy.

When the cause of the symptoms of heartburn and regurgitation were correctly attributed to gastrooesophageal reflux, ${ }^{6}$ it was assumed that this reflux was made possible by the presence of a sliding hiatal hernia. It was further assumed that, if this hernia were repaired according to the general principles of hernia repair, then the abnormal reflux would be prevented and the patient cured. Unfortunately, anatomical repair of the hiatus frequently did not prevent further reflux, however meticulously the operation was carried out and by whatever method it was performed. ${ }^{78}$

\footnotetext{
*Based on a communication read to the British Society of Gastro enterology, September 1979.

†Address for reprints: Mr R J R Goodall, St James's Hospital, Beckett Street, Leeds L59 7TF.
}

Received for publication 7 January 1980
Surgical procedures for controlling gastrooesophageal reflux aim now at the restoration of competence at the gastro-oesophageal junction by a variety of techniques. Fundoplication ${ }^{9}$ has perhaps become the most commonly used of these techniques, as it can be carried out by abdominal or thoracic approaches and gives good results. ${ }^{10}$ It can even be used when the gastro-oesophageal junction cannot be restored to the abdomen. ${ }^{11}$ However, the initial results of the Allison repair looked good, and it has been suggested that perhaps the current types of hernia repair (sic) will, like the Allison procedure fade away..$^{12}$

This study was performed, therefore, to provide objective evidence that one anti-reflux procedurethe Nissen fundoplication-achieved its aim of restoring competence to the gastro-oesophageal junction and would thus be effective in preventing further gastro-oesophageal reflux and its sequelae.

\section{Methods}

\section{SUBJECTS}

Twelve patients form the basis of this study. There were eight males and four females, whose ages ranged from 29 to 68 years (mean 51.3 years). All had severe symptoms of gastro-oesophageal reflux or its sequelae which were inadequately controlled by conservative measures. Contrast radiology was normal in seven patients. In the 


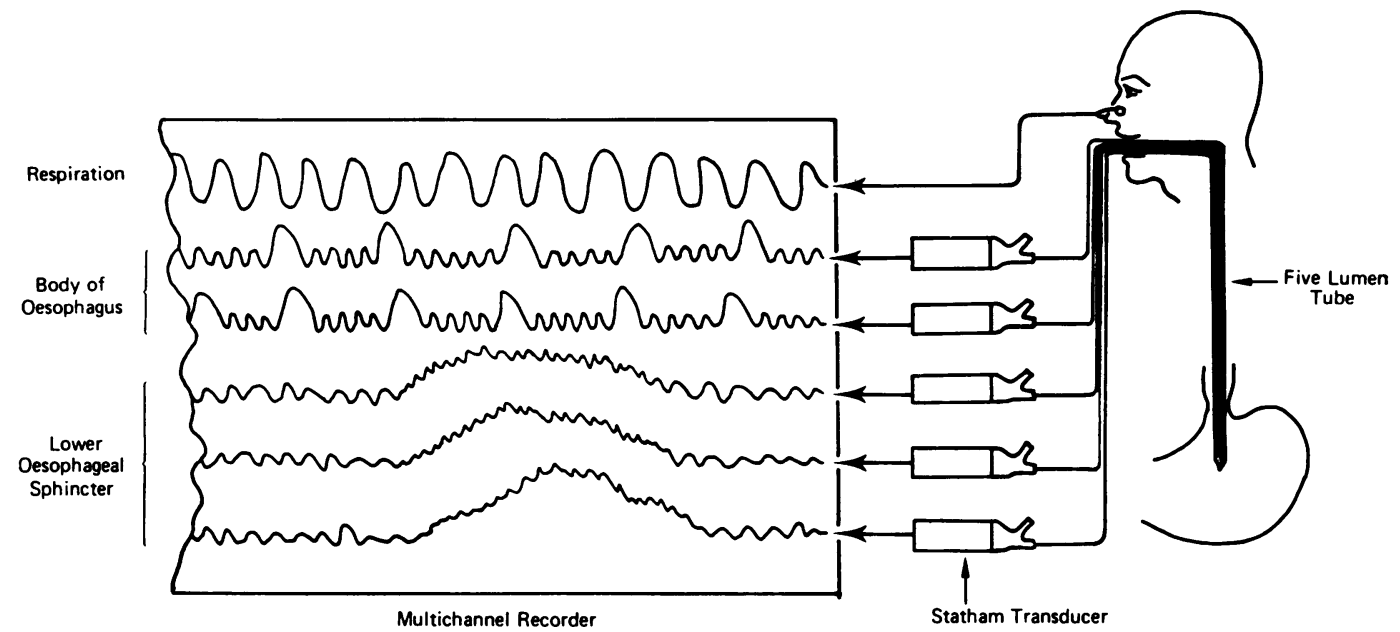

Fig. 1 Lower oesophageal manometry equipment. (Not illustrated-constant perfusion pump.)

remainder there were three reports of hiatus hernia, two of free reflux of barium, and in three a stricture was noted. All patients had fibreoptic endoscopy performed which confirmed the presence of severe oesophagitis in all and a benign reflux stricture in three. These strictures were initially dilated before medical therapy was instituted.

Informed consent was obtained in each case. All patients were studied by manometry of the lower oesophagus and by prolonged monitoring of the distal oesophageal $\mathrm{pH}$. These tests were carried out before, and three months after operation.

\section{EQUIPMENT AND TECHNIQUE}

Manometry (Fig.1)

Manometric assessment of the lower oesophageal high pressure zone (HPZ) was performed by the station pull through technique we have described, ${ }^{13}$ with one difference. The manometry tube was modified to eliminate the effect caused by the projection of a long length of catheter into the stomach. ${ }^{13} \mathrm{~A}$ five lumen tube was used which had three recording orifices situated around the circumference of the catheter $5 \mathrm{~cm}$ from the tip, and two further orifices, one $10 \mathrm{~cm}$ and the other $15 \mathrm{~cm}$ from the tip. After a four hour fast and abstinence from smoking, the manometry catheter was passed via the mouth until the distal recording orifices were in the gastric lumen. After a 10 to 15 minute rest, the tube was then withdrawn across the gastrooesophageal junction in $1 \mathrm{~cm}$ increments, with a pause between each withdrawal of at least five respiratory cycles.

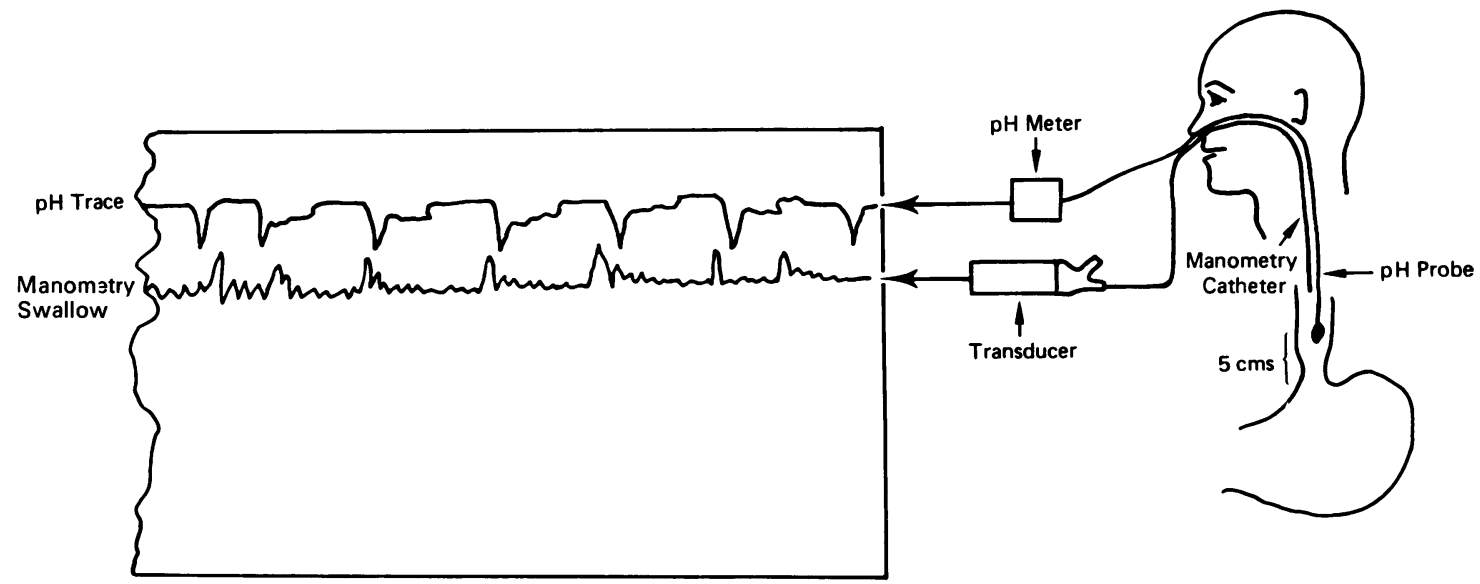

Fig. 2 Prolonged pH monitoring equipment. 
Prolonged pH monitoring (Fig. 2)

A Radiometer $\mathrm{pH}$ electrode, type GK282C, was uesd as this obviates the need for a separate reference electrode to be attached to the subject. The output from the $\mathrm{pH}$ probe was led via a $\mathrm{pH}$ meter to a Grass polygraph recorder, which provided a permanent record for subsequent analysis. Before use the apparatus was calibrated with known buffers. Attached to the $\mathrm{pH}$ probe was a fine manometry tube with a side hole placed $4 \mathrm{~cm}$ proximal to the tip of the $\mathrm{pH}$ probe. This catheter was perfused with water at $2.7 \mathrm{ml}$ per hour, and was connected via a Statham transducer to a separate channel on the Grass recorder.

After the nasopharynx had been anaesthetised with Xylocaine spray, the probe assembly was passed via the nostril into the stomach. With the subject supine the probe was withdrawn in stages to locate the pressure zone in the lower oesophagus accurately. Further withdrawal could then place the $\mathrm{pH}$ probe $5 \mathrm{~cm}$ above this pressure zone. The tube was then taped securely to the nose. The recording was continued overnight with the subject confined to the bed but free to adopt any position in bed they found comfortable. A normal hospital diet was supplied, but smoking was discouraged.

\section{ANALYSIS OF TRACES}

Manometry (Fig. 3)

As no new sphincter is created by any anti-reflux procedure, it would be incorrect in this situation to refer to a lower oesophageal sphincter or its pressure. The term high pressure zone has therefore been preferred in this paper.

The mean of 10 measurements, taken at the endexpiratory position, was determined for both the intra-gastric pressure and the maximum pressure observed in the lower oesophagus. The magnitude of the HPZ was taken as the difference between them and it has been expressed in kilopascals with millimetres of mercury in parentheses. The length of the HPZ was also measured in centimetres and its relationship to the pressure inversion point (PIP) determined. PIP occurs where the respiratory fluctuation on the trace changes from positive to negative with inspiration as the catheter is withdrawn from the abdomen into the thorax. ${ }^{14}$

\section{Prolonged $\mathrm{pH}$ monitoring}

The $\mathrm{pH}$ trace was analysed for the period $6 \mathrm{pm}$ to $6 \mathrm{am}$. Reflux was judged to have occurred when the $\mathrm{pH}$ fell below 4. Episodes of reflux lasting less than 30 seconds were ignored. In addition to the number of minutes of the test period spent with a $\mathrm{pH}$ below 4 , the number of reflux episodes was counted, the longest reflux episode was measured, and the number of reflux episodes lasting more than five minutes was noted. ${ }^{15}$

\section{STATISTICAL ANALYSIS}

The results have been expressed as means with one standard error of the mean in the text and the figures.

The values for each measured index before and after operation were compared using a paired Student's $t$ test.

\section{Results}

There were no postoperative deaths. All patients were free from reflux symptoms and none had dysphagia. Two patients had an episode of abdominal distress caused by the inability to belch or vomit - the 'gas-bloat' syndrome-after Christmas dinner, 1977. One of them had another episode after a 'good night out'. None of the patients who had a reflux stricture has required postoperative dilatation. There has therefore been no clinical indication to perform postoperative endoscopy in these patients.

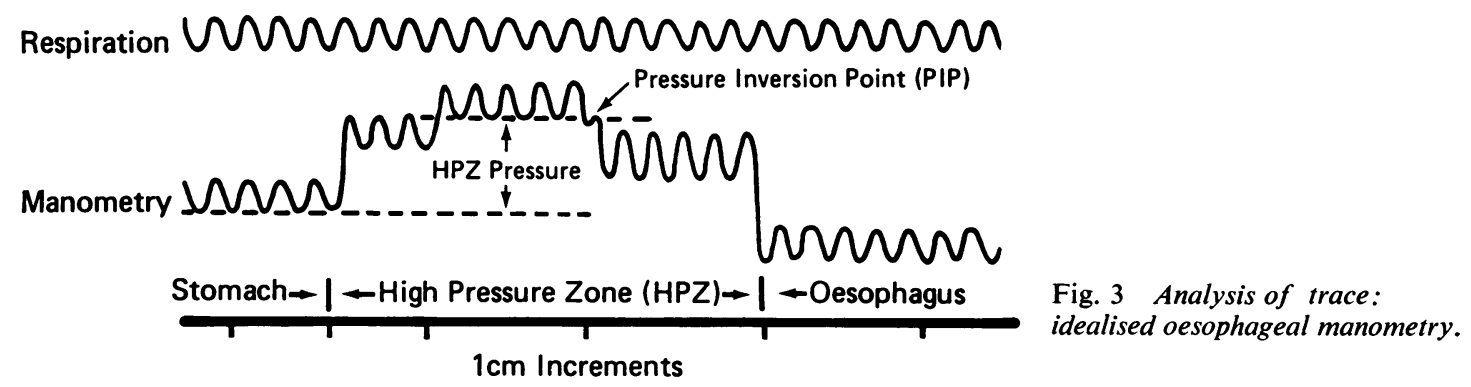



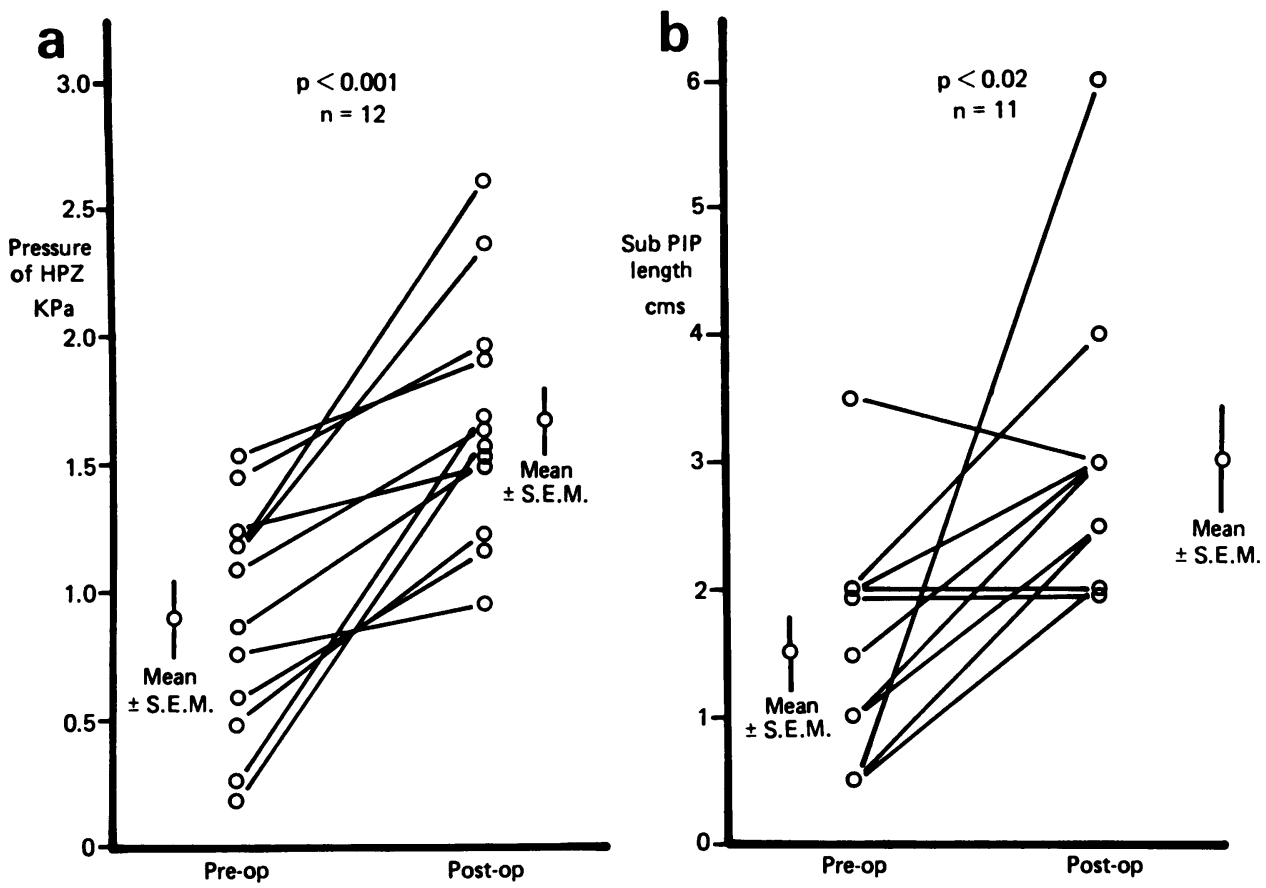

Fig. 4 Lower oesophageal manometry results. (a) HPZ pressure. (b) Sub-PIP length.

\section{ANALYSIS OF TRACES}

Manometry (Fig. 4)

The Nissen fundoplication produced a significant increase in the lower oesophageal pressure zone. The mean value for all the patients was $0.91 \mathrm{KPa} \pm$ $0.15(6.8 \mathrm{mmHg} \pm 1 \cdot 1)$ before operation and this was increased to $1.68 \mathrm{KPa} \pm 0.12(12.6 \mathrm{mmHg} \pm 0.9)$ postoperatively $(\mathrm{P}<0.001)$. More important than mean values, however, are the individual results. In all patients the pressure was greater postoperatively, such that only three patients had a pressure below $1.33 \mathrm{KPa}(10 \mathrm{mmHg})$, whereas preoperatively 10 had a value below this (Fig. 4a).

The other main effect of the fundoplication upon the HPZ was to increase the amount of the zone located below PIP. Although the total length of the zone was slightly greater postoperatively $(4.3 \mathrm{~cm}$ $\pm 0.4)$ than preoperatively $(3.4 \mathrm{~cm} \pm 0.3)$, this was not a statistically significant difference. However, the length below PIP increased from $1.5 \mathrm{~cm}( \pm 0.3)$ to $3.0 \mathrm{~cm}( \pm 0.4)$ (Fig. 4b) and this is a significant difference $(P<0.02)$. In one patient the preoperative length of the HPZ could not be accurately determined from the trace and so the postoperative value for that patient has been excluded from analysis. Only 11 pairs of figures have therefore been used.
Prolonged pH monitoring (Fig. 5)

Although $\mathrm{pH}$ monitoring was performed on all patients the results from those patients with a reflux stricture were excluded as the stricture would to an uncertain extent protect the probe from refluxed material. Postoperatively none of these three patients had recurrent dysphagia and none had any reflux whatsoever detected on their postoperative $\mathrm{pH}$ test. In the other nine patients, all four measured indices of the prolonged $\mathrm{pH}$ monitoring were improved postoperatively. The duration of reflux in the 12 hour test period fell in all patients-six of them had no reflux episode noted at all-from 98 minutes $( \pm 30)$ to 1.3 minutes $( \pm 0.9)$. This is a significant reduction $(\mathrm{P}<0.02)$ (Fig. 5a).

The number of reflux episodes also fell markedly $-17 \pm 4$ to $2 \pm 1 \quad(P<0.01)$ (Fig. 5b). The longest reflux episode recorded preoperatively was 67 minutes (mean $31 \pm 8$ ), whereas postoperatively the longest was 1.5 minutes (mean $0.4 \pm 0.2)(\mathrm{P}<0.01)$, and on average each subject had four episodes lasting over five minutes before $(3.9 \pm 1 \cdot 2)$, but there were none at all after surgery $(\mathrm{P}<0.02)$.

\section{Discussion}

The primary aim of the Nissen fundoplication, or, 
indeed, any anti-reflux procedure, is the restoration of competence at the gastro-oesophageal junction, and the reduction or repair of any sliding hiatal hernia is of lesser importance. Indeed, the Nissen fundoplication would appear to provide effective symptomatic benefit when performed intra-thoracically in cases in which the gastro-oesophageal junction cannot be reduced into the abdomen.11 It is thus apparent that judging the efficacy of any anti-reflux procedure by the radiological demonstration of the position of the gastro-oesophageal junction is questionable. ${ }^{10}$ Similarly, the visualisation of reflux of barium on fluoroscopy, which is of little significance preoperatively, ${ }^{1617}$ cannot then be used to assess the postoperative subject. DeMeester et al ${ }^{10}$ also found that an improvement in symptoms did not necessarily mean an absence of abnormal reflux as judged by $\mathrm{pH}$ monitoring.

At the present time the best method of documenting reflux is by the application of the techniques of pH monitoring in the distal oesophagus. 151819

The relative importance of the lower oesophageal HPZ in the maintenance of gastro-oesophageal competence is still disputed. Clark et al. ${ }^{16}$ although demonstrating a significant difference between the mean HPZ pressure for normal subjects and patients with reflux, have shown that there is such a wide overlap as to make pressure measurement alone of no discriminatory value. Cohen and Harris, ${ }^{16}$ on the other hand, showed clear distinction between their two groups of normal and refluxers, and Cohen and Snape ${ }^{20}$ continue to believe that manometric measurement of the HPZ can identify an incompetent gastro-oesophageal junction. Of the other factors thought to be important in the anti-reflux mechanism the two which are directly affected by the anti-reflux procedures are the length of intraabdominal oesophagus (although this is not essential for the Nissen procedure), and the acute gastrooesophageal angle. Only the former can be readily assessed, both pre and postoperatively, by manometry.

In our assessment of the Nissen fundoplication we have therefore measured by manometry those indices of the lower oesophageal HPZ which have been altered by the surgery and then determined whether these changes have been accompanied by gastro-oesophageal competence as judged by prolonged $\mathrm{pH}$ monitoring.

The manometric data show improvement in all patients. The pressure of the HPZ increased significantly above preoperative values and individual
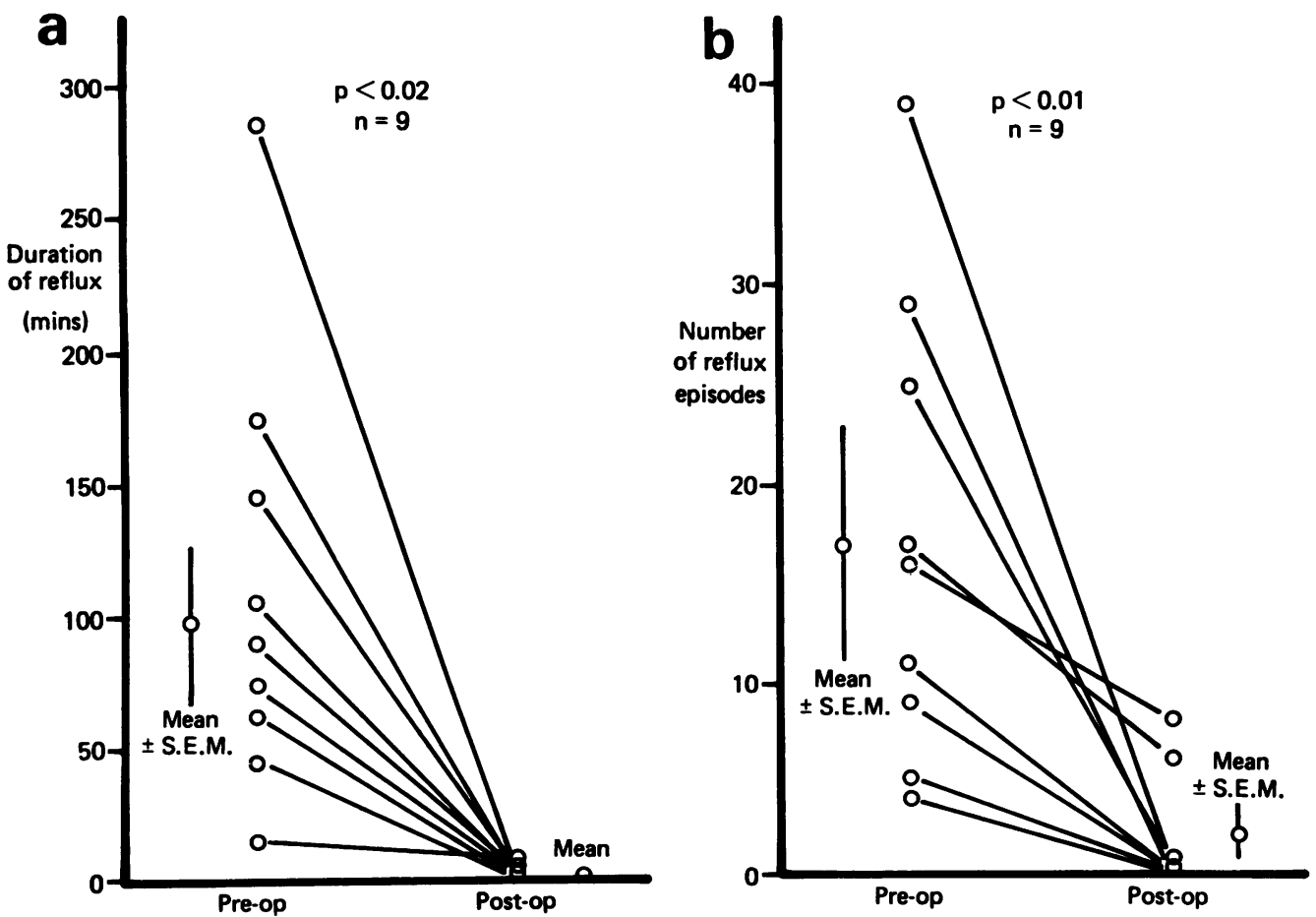

Fig. 5 Prolonged pH monitoring results. (a) Duration of reflux. (b) Number of reflux episodes. 
values were all improved. The total length of the HPZ was not significantly increased by operation but the length of the zone below PIP was significantly increased, being doubled on average. Although the reliability of PIP in identifying the diaphragmatic hiatus has been questioned, ${ }^{21}$ the exposure of a greater proportion of the HPZ to positive inspiratory pressure should contribute to improved competence at the gastro-oesophageal junction.

These findings are in accord with other published data following Nissen fundoplication. ${ }^{10}$ The values we recorded for the pressures achieved are almost exactly half those recorded by DeMeester and his colleagues. As this applies equally to both the pre and postoperative values, this difference is probably a reflection of the use of different techniques in performing the manometry. ${ }^{1322}$ In both this and DeMeester's study the effect of the operation was almost to double the mean value for the HPZ pressure. DeMeester and his colleagues also achieved a significant increase in the portion of the HPZ below PIP without increasing significantly the total length.

Preoperatively the HPZ represents the intrinsic tone of the lower oesophageal sphincter. Postoperatively the HPZ may result from other factors as well. In cats ${ }^{23}$ and humans ${ }^{24}$ the intrinsic sphincter tone can be reduced experimentally by the infusion of acid into the lower oesophagus. It is possible therefore that the prevention of reflux may allow a low sphincter pressure to improve. But there is undoubtedly a large contribution to postoperative pressure from the fundoplication itself which can be observed and measured during the procedure (personal observations).

The prolonged $\mathrm{pH}$ monitoring has confirmed the restoration of competence to the gastro-oesophageal junction achieved by the Nissen procedure. Nine of the 12 patients were found to have no reflux whatsoever in a 12 hour overnight test. The other three patients all had minimal amounts of reflux. This contrasts markedly with the gross quantities of reflux seen preoperatively in these patients, with the exception of those with stricture formation. Our results confirm the findings of DeMeester et al. ${ }^{10}$ that a Nissen fundoplication affords very good protection against further reflux as judged by prolonged $\mathrm{pH}$ monitoring.

However, these results do not indicate how the Nissen procedure achieves its effect. There is no correlation between the manometric changes either in pressure or length of the HPZ and the decrease in reflux observed either in duration or number of episodes. In other words, small increases in the pressure or sub-PIP length of the HPZ seem to be as effective in preventing excessive gastro-oesophageal reflux as larger increases in these variables. It is therefore possible that the efficacy of the fundoplication depends upon a secondary and perhaps more mechanical effect, such as the creation of a valve or alteration of the gastro-oesophageal angle at the gastro-oesophageal junction. So, although the manometric changes observed have been accompanied by an improvement in gastro-oesophageal competence, they may not be directly responsible for the reduction in gastro-oesophageal reflux.

\section{Conclusion}

We have shown that the Nissen fundoplication increases the pressure in the lower oesophageal HPZ and that it increases the amount of this zone in the abdominal cavity.

These changes are associated with the restoration of competence at the gastro-oesophageal junction and therefore the prevention of reflux of acid gastric juice into the oesophagus.

This type of study should be carried out after other anti-reflux procedures in order to document their efficacy with objective data.

\section{References}

${ }^{1}$ Lyons WS, Ellis FH, Olsen AM. The gastroesophageal 'sphincter' mechanism. A review. Proc Mayo Clin 1956; 31 : 605-14.

'Ingelfinger FJ. Esophageal motility. Physiol Rev 1958; 38: 533-84.

${ }^{3}$ Botha GSM. The gastro-oesophageal junction. Boston: Little, Brown, 1962.

${ }^{4}$ Hiebert CA, Belsey R. Incompetency of the gastric cardia without radiologic evidence of hiatal hernia. $J$ Thorac Cardiovasc Surg 1961 ; 42: 352-62.

${ }^{5}$ Cohen S., Harris LD. Does hiatus hernia affect competence of the gastroesophageal sphincter? $N$ Engl $J$ Med 1971; 284: 1053-6.

${ }^{6}$ Allison PR. Peptic ulcer of the oesophagus. $J$ Thorac Surg 1946; 15: 308-17.

${ }^{7}$ Nissen R. Gastropexy as the lone procedure in the surgical repair of hiatus hernia. Am J Surg 1956; 92 : 389-92.

${ }^{8}$ Allison PR. Hiatus hernia. A 20 year retrospective survey. Ann Surg 1973; 178: 273-6.

${ }^{9}$ Nissen R. Gastropexy and 'fundoplication' in surgical treatment of hiatal hernia. Am J Dig Dis 1961; 6: 954-61.

${ }^{10}$ DeMeester TR, Johnson LF, Kent AH. Evaluation of current operations for the prevention of gastroesophageal reflux. Ann Surg 1974; 180: 511-25. 
${ }^{11}$ Moghissi K. Conservative surgery in reflux stricture of the oesophagus associated with hiatal hernia. Brit $J$ Surg 1979; 66: 221-5.

${ }^{12}$ Ingelfinger FJ. The sphincter is a sphinx. $N$ Engl J Med 1971; 284: 1095-6.

${ }^{13}$ Hay DJ, Goodall RJR, Temple JG. The reproducibility of the station pullthrough technique for measuring lower oesophageal sphincter pressure. Brit J Surg 1979; 66: $93-7$.

${ }^{14}$ Skinner DB, DeMeester TR. Gastroesophageal reflux. Curr Probl Surg 1976; 13: 1-62.

${ }^{15}$ Johnson LF, DeMeester TR. Twenty-four-hour $\mathrm{pH}$ monitoring of the distal esophagus. A quantitative measure of gastroesophageal reflux. Am J Gastroenterol 1974; 62: 325-32.

${ }^{16}$ Clark J, Moossa AR, Skinner DB. Pitfalls in the performance and interpretation of esophageal function tests. Surg Clin N Amer 1976; 56: 29-37.

${ }^{17}$ DeMeester TR, Johnson LF. The evaluation of objective measurements of gastroesophageal reflux and their contribution to patient management. Surg Clin $N$ Amer 1976; 56: 39-53.
${ }^{18}$ Atkinson M. Standard tests and practice-II. In: Abbey Smith R, Smith RE. eds. Surgery of the oesophagus. The Coventry Conference. London: Butterworths, 1972: 125-31.

${ }^{19}$ Pope CE. Pathophysiology and diagnosis of reflux esophagitis. Gastroenterology 1976; 70: 445-454.

${ }^{20}$ Cohen S, Snape WJ Jr. The pathophysiology and treatment of gastroesophageal reflux disease. Arch Int Med 1978; 138: 1398-401.

${ }^{21}$ Harris LD, Pope CE. The pressure inversion point: its genesis and reliability. Gastroenterology 1966; 51: 641-8.

${ }^{22}$ Earlam R. Clinical tests of oesophageal function. London: Crosby Lockwood, Staples, 1976.

${ }^{23}$ Eastwood GL, Castell DO, Higgs RH. Experimental esophagitis in cats impairs lower esophageal sphincter pressure. Gastroenterology 1975; 69: 146-53.

${ }^{21}$ Waterfall WE, Lewis TD, Fox JET, Daniel EE. pH as a determinant of lower esophageal sphincter tone. (Abstract.) Gastroenterology 1979; 76: 1267. 